\title{
Density-Functional Theory with Screened van der Waals Interactions for the Modeling of Hybrid Inorganic-Organic Systems
}

\author{
Victor G. Ruiz, ${ }^{1}$ Wei Liu, ${ }^{1}$ Egbert Zojer, ${ }^{2}$ Matthias Scheffler, ${ }^{1}$ and Alexandre Tkatchenko ${ }^{1}$ \\ ${ }^{1}$ Fritz-Haber-Institut der Max-Planck-Gesellschaft, Faradayweg 4-6, 14195, Berlin, Germany \\ ${ }^{2}$ Institute of Solid State Physics, Graz University of Technology, Petersgasse 16, A-8010 Graz, Austria
}

(Received 15 November 2011; published 6 April 2012)

\begin{abstract}
The electronic properties and the function of hybrid inorganic-organic systems (HIOS) are intimately linked to their interface geometry. Here we show that the inclusion of the many-body collective response of the substrate electrons inside the inorganic bulk enables us to reliably predict the HIOS geometries and energies. This is achieved by the combination of dispersion-corrected density-functional theory (the DFT + van der Waals approach) [Phys. Rev. Lett. 102, 073005 (2009)], with the Lifshitz-Zaremba-Kohn theory for the nonlocal Coulomb screening within the bulk. Our method yields geometries in remarkable agreement $(\approx 0.1 \AA)$ with normal incidence $x$-ray standing wave measurements for the $3,4,9$, 10-perylene-tetracarboxylic acid dianhydride $\left(\mathrm{C}_{24} \mathrm{O}_{6} \mathrm{H}_{8}\right.$, PTCDA) molecule on $\mathrm{Cu}(111), \operatorname{Ag}(111)$, and $\mathrm{Au}(111)$ surfaces. Similarly accurate results are obtained for xenon and benzene adsorbed on metal surfaces.
\end{abstract}

DOI: 10.1103/PhysRevLett.108.146103

PACS numbers: 68.35. $-\mathrm{p}, 68.43 .-\mathrm{h}, 73.20 . \mathrm{Mf}$

Hybrid inorganic-organic systems (HIOS) are essential ingredients of a wide range of emerging devices. Applications in which such interfaces play a crucial role include (opto)-electronics (e.g., transistors, light-emitting diodes), organic photovoltaics, and sensors [1]. The interface geometry of HIOS plays a crucial role in the determination of their electronic properties, and the accurate prediction of interface structure and stability is essential for controlling the function and quality of these highly sought-after technologies.

Within the possible variety of HIOS, 3,4,9,10-perylenetetracarboxylic acid dianhydride $\left(\mathrm{C}_{24} \mathrm{O}_{6} \mathrm{H}_{8}\right.$, PTCDA) on coinage metals is one of the best experimentally and theoretically characterized systems. The adsorption geometry of PTCDA on $\mathrm{Cu}(111), \operatorname{Ag}(111)$, and $\mathrm{Au}(111)$, has been accurately determined by means of the normal incidence x-ray standing wave (NIXSW) technique [2-4], making these systems a suitable choice for testing the predictive power of theoretical methods.

The reliable prediction of the equilibrium structure and dynamic properties of HIOS from first principles represent a great challenge to the state-of-the-art theoretical methods due to the interplay of covalent interactions, electron transfer processes, van der Waals (vdW) interactions, and Pauli repulsion. In particular, vdW interactions are fundamental in determining the structure and stability of organic molecules on solid surfaces [5-11]. Romaner et al. studied the adsorption of PTCDA on coinage metals [12], and

Published by the American Physical Society under the terms of the Creative Commons Attribution 3.0 License. Further distribution of this work must maintain attribution to the author(s) and the published article's title, journal citation, and DOI. concluded that a more accurate approach to include vdW interactions is needed.

To illustrate the problem, we start by comparing the performance of existing theoretical methods for the interaction of PTCDA with $\operatorname{Ag}(111)$ in Fig. 1. The average adsorption distance $(2.86 \AA)$ is reliably known from a number of NIXSW studies for the ordered monolayer at room temperature (see Ref. [4] and references therein). There is also an estimate of the adsorption energy of $2.4 \mathrm{eV}$ by extrapolation from the 1,4,5,8-naphtalenetetracarboxylic acid dianhydride $\left(\mathrm{C}_{14} \mathrm{O}_{6} \mathrm{H}_{4}\right.$, NTCDA)

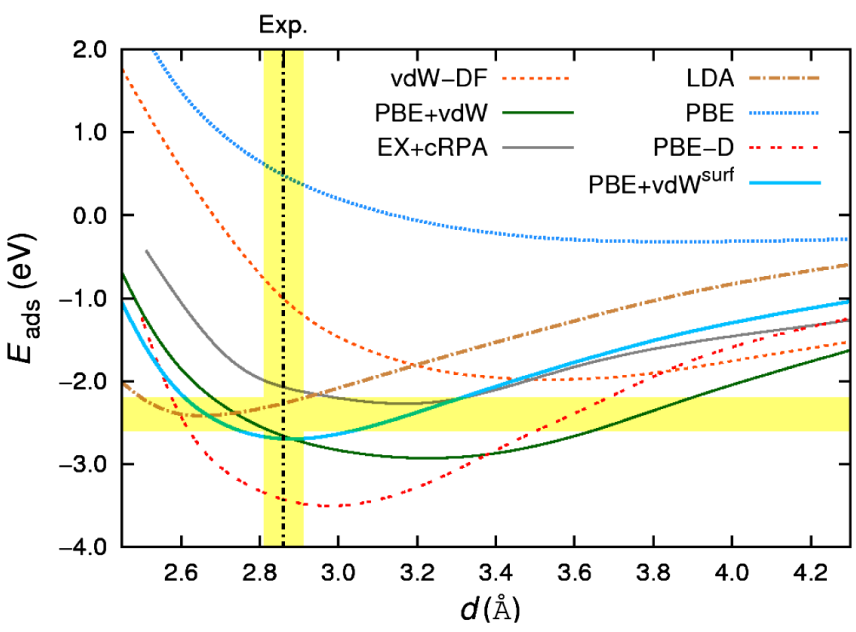

FIG. 1 (color online). Adsorption energy $E_{\text {ads }}$ as a function of vertical distance $d$ for PTCDA on $\mathrm{Ag}(111)$ employing different theoretical approaches. The estimated adsorption energy for the system $(2.4 \pm 0.1 \mathrm{eV} \mathrm{[10])} \mathrm{and} \mathrm{the} \mathrm{experimental} \mathrm{adsorption}$ distance $(2.86 \pm 0.05 \AA[4])$ are indicated by shaded intervals. These error bars correspond to typical experimental error estimates. 
molecule, which is closely related to PTCDA and experimentally accessible to temperature-programmed desorption $[10,13]$. Using standard density-functional theory (DFT) with the Perdew-Burke-Ernzerhof (PBE) [14] functional results in no visible minimum in the binding curve [10]. The local-density approximation (LDA) underestimates the binding distance $(\approx 2.65 \AA)$, although it (fortuitously) yields a better adsorption energy [15]. The inclusion of $\mathrm{vdW}$ interactions using the vdW-DF nonlocal functional [16] leads to far off results both for the binding distance and the binding energy $[10,12]$. Interatomic pairwise PBE-D correction by Grimme [17] appears to be closer to the experimental binding distance; the calculated binding energy is, however, by $1 \mathrm{eV}$ larger than the above mentioned estimate. The interatomic pairwise $\mathrm{PBE}+\mathrm{vdW}$ correction scheme [18], where the vdW coefficients and radii are determined nonempirically from the electron density, leads to a better prediction for the energy but it still overestimates the equilibrium distance by about $0.25 \AA$. Even the computationally most expensive calculations using exact exchange with electron correlation treated in the random-phase approximation (with some additional approximations to make these calculations feasible) yield a $0.2 \AA$ overestimation in the equilibrium distance of PTCDA on $\mathrm{Ag}(111)$ [19].

In this Letter, we propose a method that extends standard pairwise vdW corrections [17,18] to the modeling of adsorbates on surfaces. Here, this is achieved by combining the DFT + vdW scheme [18] with the LifshitzZaremba-Kohn (LZK) theory for the vdW interaction between an atom and a solid surface [20,21]. In our approach (DFT $\left.+\mathrm{vdW}^{\text {surf }}\right)$, the $\mathrm{vdW}$ energy correction to the DFT total energy is given by a sum of $C_{6}^{a b} R_{a b}^{-6}$ terms, where $R_{a b}$ are the distances between atoms $a$ and $b$, in analogy to the DFT-D and DFT $+\mathrm{vdW}$ methods. By employing the LZK theory, however, we include the many-body collective response (screening) of the substrate electrons in the determination of the $C_{6}$ coefficients and vdW radii, going effectively beyond the pairwise description. Interface polarization effects are accounted for via the inclusion of semilocal hybridization due to the dependence of the $C_{6}^{a b}$ interatomic coefficients on the electron density in the DFT + vdW method. We show that nonlocal screening reduces the vdW $C_{6}$ coefficients by up to a factor of 4 for coinage metals. As indicated by the solid blue (light gray) line in Fig. 1 for the PTCDA/Ag(111) interface, $\mathrm{DFT}+\mathrm{vdW}{ }^{\text {surf }}$ indeed provides the most accurate results for the binding distance and energy when compared to all other theoretical methods. Similarly accurate results are found for PTCDA adsorbed on other coinage metals as well as for Xe and benzene on a range of metallic surfaces.

Beyond the region of orbital overlap, the vdW interaction of an atom $a$ with a solid $B$ is given by $E_{\mathrm{vdW}} \simeq$ $-C_{3}^{a B} /\left(Z-Z_{0}\right)^{3}$, where $Z$ is the distance from the $\mathrm{vdW}$ image plane $Z_{0}$, and the $C_{3}^{a B}$ is the corresponding vdW interaction coefficient [20-22]. Zaremba and Kohn derived this formula starting from quantum mechanics, obtaining the following expression for the $C_{3}^{a B}$ coefficient $[20,21]$

$$
C_{3}^{a B}=\frac{\hbar}{4 \pi} \int_{0}^{\infty} d \omega \alpha(i \omega) \frac{\varepsilon_{B}(i \omega)-1}{\varepsilon_{B}(i \omega)+1},
$$

where $\alpha(i \omega)$ is the polarizability of the atom $a$, and $\varepsilon_{B}(i \omega)$ is the dielectric function of the solid $B$. Equation (1) incorporates screening effects inside the bulk as it is evident from its dependence on the dielectric function $\varepsilon_{B}(i \omega)$.

A convenient approach to recover the LZK formula $[22,23]$, is to determine the $\mathrm{vdW}$ interaction of an atom $a$ with a solid $B$ by a summation of $-C_{6}^{a b} / R_{a b}^{6}$ pair potentials between the atom $a$ and the atoms $b$ in the infinite halfspace of the solid $B$. In this case, the image-plane position is given as $Z_{0}=d / 2$, where $d$ is the interlayer distance, and the $C_{3}^{a B}$ coefficient is obtained as

$$
C_{3}^{a B}=n_{s}\left(\frac{\pi}{6}\right) C_{6, \mathrm{LZK}}^{a b},
$$

where $n_{s}$ is the number of atoms per unit volume in the bulk.

Combining Eqs. (1) and (2), the screened $C_{6, \mathrm{LZK}}^{a b}$ coefficient is derived. It effectively "inherits" the many-body screening effects contained in $C_{3}^{a B}$ from Eq. (1). The next step is to disentangle the heteronuclear $C_{6, \mathrm{LZK}}^{a b}$ interaction coefficient into homonuclear $C_{6}^{a a}$ and $C_{6, \mathrm{LZK}}^{b b}$ coefficients. For this purpose, we adopt a Padé-approximant model for the atomic polarizability as in the DFT + vdW method [18]. The Padé-approximant model allows us to perform analytic frequency integration, leading to a simple combination rule for $C_{6, \mathrm{LZK}}^{a b}$

$$
C_{6, \mathrm{LZK}}^{a b}=\frac{2 C_{6}^{a a} C_{6, \mathrm{LZK}}^{b b}}{\frac{\alpha_{\mathrm{LZK}}^{b}}{\alpha_{0}^{a}} C_{6}^{a a}+\frac{\alpha_{0}^{a}}{\alpha_{\mathrm{LZK}}^{b}} C_{6, \mathrm{LZK}}^{b b}} .
$$

The $\alpha_{0}^{a}$ and $\alpha_{\mathrm{LZK}}^{b}$ correspond to the adsorbate atom and the effective "atom-in-a-solid" static polarizabilities, respectively. In the context of LZK theory, the frequencydependent polarizability of the adsorbed atom is equivalent to the one of the free atom in the gas phase. Since accurate $\alpha_{0}^{a}$ and $C_{6}^{a a}$ values are known for the free atoms [24], the two parameters to be determined in Eq. (3) are $\alpha_{\mathrm{LZK}}^{b}$ and $C_{6, \mathrm{LZK}}^{b b}$. We obtained them by solving a system of two equations [Eq. (3)] using any two atoms from the list: $\mathrm{H}$, $\mathrm{C}, \mathrm{Ne}, \mathrm{Ar}$, and $\mathrm{Kr}$ for a given solid $B$. The dielectric function $\varepsilon_{B}(i \omega)$ was calculated in terms of the absorptive part of the dielectric function $\varepsilon_{2}$ at real frequencies by the Kramers-Kronig relation. The data were taken from the reflection energy-loss spectroscopy (REELS) experiments by Werner et al. [25]. The LZK dispersion coefficients along with $\alpha_{\mathrm{LZK}}^{b}$ for an atom $b$ inside the bulk corresponding to $\mathrm{Cu}, \mathrm{Ag}, \mathrm{Au}, \mathrm{Pd}$, and Pt metals, obtained with the above procedure, are shown in Table I. For comparison, the 
TABLE I. Screened $C_{6}$ coefficients (hartree $\cdot \mathrm{bohr}^{6}$ ), polarizability (in bohr ${ }^{3}$ ), and vdW radii (in bohr). Free atomic parameters as used in the DFT $+\mathrm{vdW}$ method are also shown for comparison.

\begin{tabular}{lcccccc}
\hline \hline Substrate & $C_{6, \text { LZK }}^{b b}$ & $\alpha_{\text {LZK }}^{b}$ & $R_{\text {LZK }}^{b}$ & $C_{6, \text { free }}^{b b}$ & $\alpha_{\text {free }}^{b}$ & $R_{\text {free }}^{b}$ \\
\hline $\mathrm{Cu}$ & 59 & 10.9 & 2.40 & 253 & 42.0 & 3.76 \\
$\mathrm{Ag}$ & 122 & 15.4 & 2.57 & 339 & 50.6 & 3.82 \\
$\mathrm{Au}$ & 134 & 15.6 & 2.91 & 298 & 36.5 & 3.86 \\
$\mathrm{Pd}$ & 102 & 13.9 & 3.06 & 158 & 23.7 & 3.66 \\
$\mathrm{Pt}$ & 120 & 14.5 & 2.80 & 347 & 39.7 & 3.92 \\
\hline \hline
\end{tabular}

free atomic parameters are also displayed. The screened dispersion coefficients for atoms in the bulk are reduced by a factor of 1.5, 2, and 4 for $\mathrm{Pd}, \mathrm{Au}$, and $\mathrm{Cu}$, respectively, and 3 for $\mathrm{Ag}$ and $\mathrm{Pt}$ compared to their free atom counterparts. This clearly illustrates the sensitive dependence of the dielectric screening on the inorganic substrate. We note that both LZK parameters are essentially invariant to the nature of the adsorbed atom. Thus, they can be considered as intrinsic properties of the bulk.

The LZK theory is exact for atom-surface distances beyond orbital overlap. However, it does not include effects due to rapid spatial variations (interface polarization) in the dielectric function close to the surface. We effectively include these contributions through the linkage of the LZK theory with the DFT $+\mathrm{vdW}$ method. Once the $\alpha_{\mathrm{LZK}}^{b}, C_{6, \mathrm{LZK}}^{b b}$, and $R_{\mathrm{LZK}}^{b}$ are computed, the reference $\mathrm{DFT}+\mathrm{vdW}^{\text {surf }}$ polarizability, $C_{6}$, and vdW radius for the bulk atoms are set to those values. Nevertheless, these parameters change with the definition of a dimensionless effective volume $v_{\text {eff }}^{i}$ for species $i$, in terms of the Hirshfeld partitioning of the electron density [26,27],

$$
v_{\mathrm{eff}}^{i}=\left(\frac{\int r^{3} w_{i}(\mathbf{r}) n(\mathbf{r}) d^{3} \mathbf{r}}{\int r^{3} n_{i}^{\mathrm{ref}}(\mathbf{r}) d^{3} \mathbf{r}}\right)
$$

where $w_{i}(\mathbf{r})$ is the Hirshfeld atomic partitioning weight of the species $i, r^{3}$ is the cube of the distance from the nucleus of an atom $i, n(\mathbf{r})$ is the total electron density, and $n_{i}^{\text {ref }}(\mathbf{r})$ is the reference electron density. For the solid, the reference corresponds to the spherical electron density of an atom in the bulk, and for a molecule, it corresponds to the free atom electron density. The effective $C_{6, \text { eff }}^{i i}$ coefficient is determined as $C_{6, \text { eff }}^{i i}=\left(v_{\text {eff }}^{i}\right)^{2} C_{6 \text {,ref }}^{i i}$, the effective polarizability as $\alpha_{\mathrm{eff}}^{i}=v_{\mathrm{eff}}^{i} \alpha_{\mathrm{ref}}^{i}$, and the effective vdW radius, which determines the onset of the damping function, is calculated as $R_{\text {eff }}^{i}=\left(\alpha_{\text {eff }}^{i} / \alpha_{\text {ref }}^{i}\right)^{1 / 3} R_{\text {ref }}^{i}$ [18]. Significant interface polarization is observed in the DFT $+\mathrm{vdW}^{\text {surf }}$ method when organic molecules are adsorbed on metals. For example, for the PTCDA molecule located at the experimental equilibrium position on $\operatorname{Ag}(111)$, the molecular $C_{6 \text {,eff }}$ coefficient is increased by $9 \%$ compared to the gas-phase PTCDA, while for the surface atoms it is decreased by $4 \%$ compared to the atoms inside the bulk $\left(C_{6, \text { ref }}\right)$.
To further benchmark the DFT $+\mathrm{vdW}^{\text {surf }}$ approach, we next calculated the binding energy curves for a rigid PTCDA molecule on $\mathrm{Cu}(111)$ and $\mathrm{Au}(111)$ surfaces. These are shown together with the experimentally determined average adsorption distances in Fig. 2 (Calculation details are included in the Supplemental Material [28].) The data for PTCDA on $\operatorname{Ag}(111)$ from Fig. 1 is also included for the sake of comparison. The first interesting observation comes from comparing the PBE $+\mathrm{vdW}$ and $\mathrm{PBE}+\mathrm{vdW}^{\text {surf }}$ binding curves for PTCDA/Ag(111) in Fig. 1. The LZK correction leads to a reduced $C_{6}$ coefficient (see Table I), and yields significantly smaller longrange vdW energy. However, the vdW radii of $\mathrm{Ag}(111)$ atoms are also reduced, amounting to a larger vdW contribution at shorter range. These two effects modify the binding curve for PTCDA/Ag(111), leading to a reduced adsorption energy and a reduced adsorption distance compared to PBE $+\mathrm{vdW}$. The same conclusions hold for the other coinage metals. As shown in Fig. 2, the equilibrium distances agree very well with the NIXSW results and also the adsorption energies reproduce the trend in the binding strength inferred from experiment: $E_{\text {ads }}(\mathrm{Cu})>E_{\text {ads }}(\mathrm{Ag})>$ $E_{\text {ads }}(\mathrm{Au})[4]$.

It is well known that the PTCDA molecule is significantly distorted upon adsorption on $\operatorname{Ag}(111)$ and $\mathrm{Cu}(111)$. Seeking to understand whether PBE $+\mathrm{vdW}^{\text {surf }}$ can also accurately reproduce the vertical distortion, we carried out structural relaxations of the PTCDA/Me(111) systems, where we allowed the atoms in the PTCDA molecules and in the top metal layer to relax. Figure 3 shows the

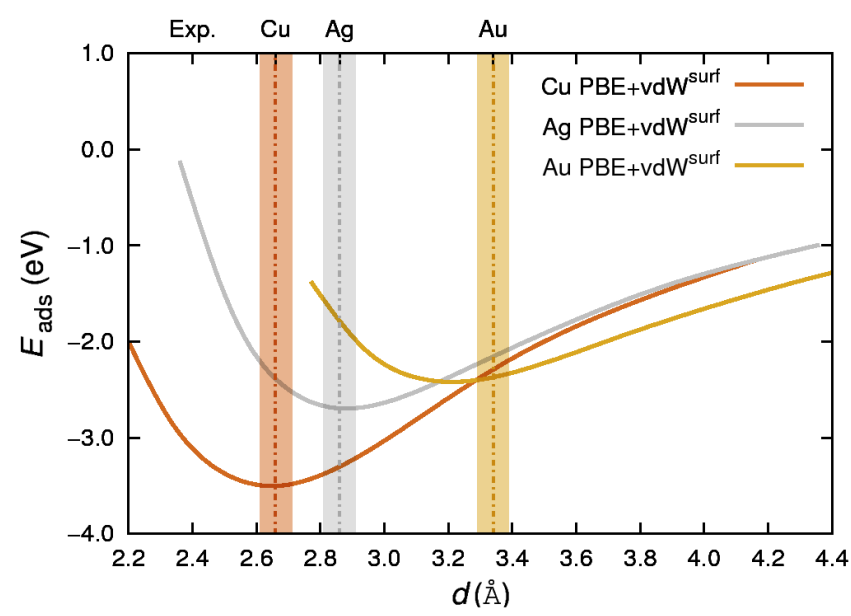

FIG. 2 (color online). Adsorption energy $E_{\text {ads }}$ as a function of vertical distance $d$ for PTCDA on $\mathrm{Cu}(111), \operatorname{Ag}(111)$ and $\mathrm{Au}(111)$ employing the DFT $+\mathrm{vdW}^{\text {surf }}$ method. The distance $d$ is evaluated with respect to the position of the unrelaxed topmost metal layer. The experimental adsorption distances are $2.66 \pm 0.05,2.86 \pm 0.05$, and $3.34 \pm 0.05 \AA$ corresponding to $\mathrm{Cu}$ [2], Ag [4], and Au [3], respectively. They are indicated by shaded regions. Calculation details are included in the Supplemental Material [28]. 
(a) PTCDA/Cu(111)
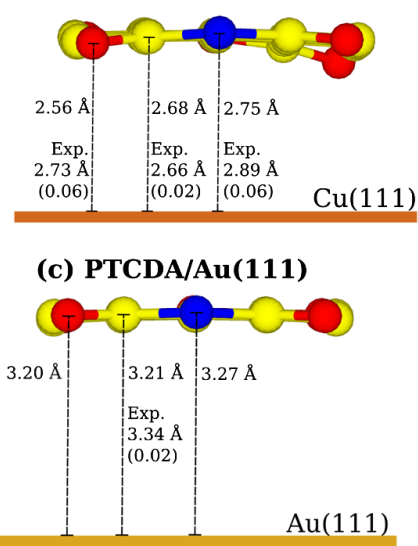

(b) PTCDA/Ag(111)
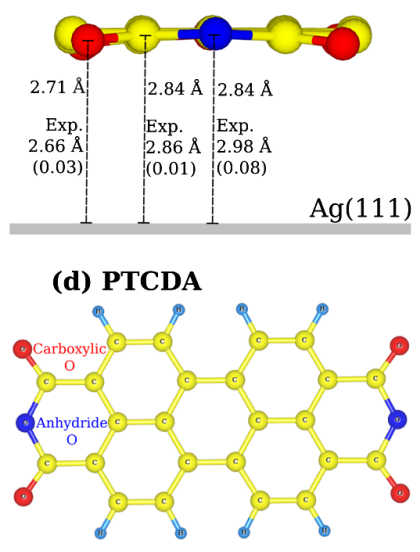

(d) PTCDA

FIG. 3 (color online). Comparison between the experimental binding distances [3,4] (with typical error bars shown in parentheses) and the calculated distances in the present work for PTCDA on $\mathrm{Cu}(111)$ (a), $\operatorname{Ag}(111)$ (b) and $\mathrm{Au}(111)$ (c). The distances are measured as an average over the constituent atoms of the two molecules in the unit cell (carbon, anhydride oxygen, and carboxylic oxygen) with respect to the unrelaxed topmost metallic layer. The structure of PTCDA is also displayed in (d). PTCDA/Cu(111) shows a distortion in the carboxylic oxygen atom, which is in agreement with a low coherent fraction for the carboxylic oxygen atom as reported for the NIXSW experiment in [2]. Calculation details are included in the Supplemental Material [28].

average bonding distances of the carbon atoms and the carboxylic and anhydride oxygens for the relaxed geometries and compares them to experimental data where available. The adsorption distance of the $\mathrm{C}$ backbone after relaxation agrees remarkably well with the experimental data to better than $0.1 \AA$ for $\mathrm{Cu}(111)$ and $\mathrm{Ag}(111)$, while an underestimation of $\approx 0.1 \AA$ is observed for $\mathrm{Au}(111)$. The slightly larger discrepancy observed for Au can be attributed to the $\mathrm{Au}(111)$ surface reconstruction [29,30]. The position of the carboxylic oxygen $\left(\mathrm{O}_{c}\right)$ for PTCDA/ $\operatorname{Ag}(111)$ agrees with experiment to better than $0.1 \AA$. Our calculations reproduce a distortion in the $\mathrm{O}_{c}$ of $0.13 \AA$ with respect to the $\mathrm{C}$ backbone due to the attraction to the surface, while the position of the anhydride oxygen $\left(\mathrm{O}_{a}\right)$ is underestimated by around $0.15 \AA$. With respect to PTCDA/Cu(111), we observe that the oxygen atoms are placed at an averaged position of $2.62 \AA$; i.e., they reside below the $\mathrm{C}$ backbone, opposite to the findings of Gerlach et al. [2]. In addition, the $\mathrm{O}_{c}$ is located below the $\mathrm{C}$ backbone, while the $\mathrm{O}_{a}$ is located above it. Our calculations for different monolayer structures show that the vertical position of the oxygen atoms in PTCDA/Cu(111) is sensitive to the lateral placement of the molecules in the unit cell, and we will present further analysis in forthcoming work [31]. Nonetheless, the overall agreement between the geometries derived from NIXSW data and DFT $+\mathrm{vdW}^{\text {surf }}$ is within $0.1 \AA$.
TABLE II. Comparison of equilibrium distance $d_{\mathrm{Ads}-\mathrm{Sub}}$ and adsorption energy between $\mathrm{PBE}+\mathrm{vdW}^{\text {surf }}$ and experiment for $\mathrm{Xe} / \mathrm{Me}(111)$ [32,33] and $\mathrm{C}_{6} \mathrm{H}_{6} / \mathrm{Me}(111)$ systems [34-39]. Except for $\mathrm{C}_{6} \mathrm{H}_{6} / \mathrm{Pt}(111)$, we are not aware of experimental results for the equilibrium distance of the $\mathrm{C}_{6} \mathrm{H}_{6} / \mathrm{Me}(111)$ systems. For the $\mathrm{Xe} / \mathrm{Me}(111)$ systems, we report the $d_{\mathrm{Ads}-\mathrm{Sub}}$ distance for the top adsorption site. The distances are referenced to the relaxed topmost metallic layer. Calculation details are included in the Supplemental Material [28].

\begin{tabular}{lcccc}
\hline \hline Substrate & \multicolumn{2}{c}{$d_{\text {Ads-Sub }}[\AA]$} & \multicolumn{2}{c}{$E_{\text {ads }}[\mathrm{eV}]$} \\
& This work & Exp. & This work & Exp. \\
\hline $\mathrm{Xe} / \mathrm{Cu}$ & 3.46 & $3.60 \pm 0.08$ & 0.24 & $0.17-0.20$ \\
$\mathrm{Xe} / \mathrm{Ag}$ & 3.57 & $3.60 \pm 0.05$ & 0.21 & $0.20-0.23$ \\
$\mathrm{Xe} / \mathrm{Pt}$ & 3.46 & $3.40 \pm 0.10$ & 0.20 & $0.26-0.28$ \\
$\mathrm{Xe} / \mathrm{Pd}$ & 3.10 & $3.07 \pm 0.06$ & 0.29 & $0.31-0.33$ \\
$\mathrm{C}_{6} \mathrm{H}_{6} / \mathrm{Cu}$ & 2.68 & - & 0.91 & $0.68-0.81$ \\
$\mathrm{C}_{6} \mathrm{H}_{6} / \mathrm{Ag}$ & 2.93 & - & 0.81 & $0.66-0.80$ \\
$\mathrm{C}_{6} \mathrm{H}_{6} / \mathrm{Pt}$ & 2.08 & $2.02 \pm 0.02$ & 2.18 & $1.84-2.25$ \\
$\mathrm{C}_{6} \mathrm{H}_{6} / \mathrm{Pd}$ & 2.10 & - & 2.11 & - \\
\hline \hline
\end{tabular}

In order to test the applicability of the DFT $+\mathrm{vdW}^{\text {surf }}$ method to systems other than PTCDA/Me(111), we calculated the relaxed geometry for $\mathrm{Xe}$ and benzene on a variety of metallic surfaces. Also for these systems, the DFT + $\mathrm{vdW}^{\text {surf }}$ approach leads to a good agreement in the adsorption distances and interaction energies compared to available data as shown in Table II. In the case of Xe/Me(111), we find that the top and fcc hollow adsorption sites are practically degenerate. For $\mathrm{Xe} / \mathrm{Pt}(111)$, measurements show that, at low coverage, the diffussion barrier for lateral movement of the $\mathrm{Xe}$ atoms on the surface is less than $10 \mathrm{meV}$ [40]. We report the Xe-surface distance for the top site because it is experimentally determined as the most stable one. For $\mathrm{Xe} / \mathrm{Pt}(111)$, the $\mathrm{PBE}+\mathrm{vdW}$ surf method also yields an accurate vibrational energy of $3.4 \mathrm{meV}$ compared to the experimental value of $3.7 \mathrm{meV}$ [41]. Detailed analysis of this problem will be presented in a forthcoming publication [42].

In summary, we have combined the DFT $+\mathrm{vdW}$ method with the Lifshitz-Zaremba-Kohn theory, leading to a promising method (DFT $+\mathrm{vdW}^{\text {surf }}$ ) that can accurately describe the structure and energetics of HIOS. For nonmetals, the DFT $+\mathrm{vdW}^{\text {surf }}$ theory is equivalent to using screened reference parameters for an "atom in a solid" as recently presented by some of us [43]. The DFT $+\mathrm{vdW}^{\text {surf }}$ method has the same cost as the underlying DFT calculation, and does not depend on the nature of the surface, being equally applicable to insulators, semiconductors, and metals.

We are grateful for support from the FP7 Marie Curie Actions of the European Commission, via the Initial Training Network SMALL (MCITN-238804). A. T. is supported by a grant from the European Research Council (ERC Starting Grant VDW-CMAT). The authors 
acknowledge Dr. Lorenz Romaner and Dr. Oliver Hofmann for helpful discussions.

[1] L. Kronik and N. Koch, MRS Bull. 35, 417 (2010).

[2] A. Gerlach, S. Sellner, F. Schreiber, N. Koch, and J. Zegenhagen, Phys. Rev. B 75, 045401 (2007).

[3] S. K. M. Henze, O. Bauer, T.-L. Lee, M. Sokolowski, and F.S. Tautz, Surf. Sci. 601, 1566 (2007).

[4] A. Hauschild, R. Temirov, S. Soubatch, O. Bauer, A. Schöll, B. C. C. Cowie, T.-L. Lee, F. S. Tautz, and M. Sokolowski, Phys. Rev. B 81, 125432 (2010).

[5] N. Atodiresei, V. Caciuc, P. Lazić, and S. Blügel, Phys. Rev. Lett. 102, 136809 (2009).

[6] G. Mercurio et al., Phys. Rev. Lett. 104, 036102 (2010).

[7] D. Stradi et al., Phys. Rev. Lett. 106, 186102 (2011).

[8] T. Olsen, J. Yan, J. J. Mortensen, and K. S. Thygesen, Phys. Rev. Lett. 107, 156401 (2011).

[9] E. McNellis, Ph.D. thesis, Fritz-Haber-Institut der MPG, 2010.

[10] A. Tkatchenko, L. Romaner, O. T. Hofmann, E. Zojer, C. Ambrosch-Draxl, and M. Scheffler, MRS Bull. 35, 435 (2010).

[11] M.-T. Nguyen, C. A. Pignedoli, M. Treier, R. Fasel, and D. Passerone, Phys. Chem. Chem. Phys. 12, 992 (2010).

[12] L. Romaner, D. Nabok, P. Puschnig, E. Zojer, and C. Ambrosch-Draxl, New J. Phys. 11, 053010 (2009).

[13] U. Stahl, D. Gador, A. Soukopp, R. Fink, and E. Umbach, Surf. Sci. 414, 423 (1998).

[14] J. Perdew, K. Burke, and M. Ernzerhof, Phys. Rev. Lett. 77, 3865 (1996).

[15] M. Rohlfing, R. Temirov, and F. Tautz, Phys. Rev. B 76, 115421 (2007).

[16] M. Dion, H. Rydberg, E. Schröder, D. C. Langreth, and B. I. Lundqvist, Phys. Rev. Lett. 92, 246401 (2004).

[17] S. Grimme, J. Comput. Chem. 27, 1787 (2006).

[18] A. Tkatchenko and M. Scheffler, Phys. Rev. Lett. 102, 073005 (2009).

[19] M. Rohlfing and T. Bredow, Phys. Rev. Lett. 101, 266106 (2008).

[20] E. M. Lifshitz, Sov. Phys. JETP 2, 73 (1956).

[21] E. Zaremba and W. Kohn, Phys. Rev. B 13, 2270 (1976).

[22] L.W. Bruch, M.W. Cole, and E. Zaremba, Physical Adsorption: Forces and Phenomena (Oxford University Press, Oxford, 1997).
[23] S. H. Patil, K. T. Tang, and J. P. Toennies, J. Chem. Phys. 116, 8118 (2002).

[24] X. Chu and A. Dalgarno, J. Chem. Phys. 121, 4083 (2004).

[25] W. Werner, K. Glantschnig, and C. Ambrosch-Draxl, J. Phys. Chem. Ref. Data 38, 1013 (2009).

[26] F.L. Hirshfeld, Theor. Chim. Acta 44, 129 (1977).

[27] E. R. Johnson and A.D. Becke, J. Chem. Phys. 123, 024101 (2005).

[28] See Supplemental Material at http://link.aps.org/ supplemental/10.1103/PhysRevLett.108.146103 for details of the DFT calculations and the employed structures for HIOS

[29] S. Mannsfeld, M. Toerker, T. Schmitz-Hübsch, F. Sellam, T. Fritz, and K. Leo, Org. Electron. 2, 121 (2001).

[30] L. Killian, E. Umbach, and M. Sokolowski, Surf. Sci. 600, 2633 (2006).

[31] V. G. Ruiz, D. Egger, L. Romaner, E. Zojer, M. Scheffler, and A. Tkatchenko (to be published).

[32] R. D. Diehl, Th. Seyller, M. Caragiu, G. S. Leatherman, N. Ferralis, K. Pussi, P. Kaukasoina, and M. Lindroos, J. Phys. Condens. Matter 16, S2839 (2004).

[33] G. Vidali, G. Ihm, H-Y. Kim, and M. W. Cole, Surf. Sci. Rep. 12, 135 (1991).

[34] S. Lukas, S. Vollmer, G. Witte, and Ch. Wöll, J. Chem. Phys. 114, 10123 (2001).

[35] M. Xi, M. X. Yang, S. K. Jo, B. E. Bent, and P. Stevens, J. Chem. Phys. 101, 9122 (1994).

[36] R. Caputo, B. P. Prascher, V. Staemmler, P. S. Bagus, and C. Wöll, J. Phys. Chem. A 111, 12778 (2007).

[37] J. Jalkanen and F. Zerbetto, J. Phys. Chem. B 110, 5595 (2006).

[38] J. M. Gottfried, E. K. Vestergaard, P. Bera, and C. T. Campbell, J. Phys. Chem. B 110, 17539 (2006).

[39] A. Wander, G. Held, R. Q. Hwang, G. S. Blackman, M. L. $\mathrm{Xu}$, P. de Andres, M. A. Van Hove, and G. A. Somorjai, Surf. Sci. 249, 21 (1991).

[40] J. Ellis, A. P. Graham, and J. P. Toennies, Phys. Rev. Lett. 82, 5072 (1999).

[41] L. W. Bruch, A. P. Graham, and J. P. Toennies, Mol. Phys. 95, 579 (1998).

[42] W. Liu et al. (to be published).

[43] G. X. Zhang, A. Tkatchenko, J. Paier, H. Appel, and M. Scheffler, Phys. Rev. Lett. 107, 245501 (2011). 